\title{
Real-time monitoring of ubiquitination in living cells by BRET
}

Julie Perroy ${ }^{1-3}$, Stephanie Pontier ${ }^{1,3}$, Pascale G Charest ${ }^{1}$, Muriel Aubry ${ }^{1} \&$ Michel Bouvier $^{1}$

\begin{abstract}
Ubiquitin has emerged as an important regulator of protein stability and function in organisms ranging from yeast to mammals. The ability to detect in situ changes in protein ubiquitination without perturbing the physiological environment of cells would be a major step forward in understanding the ubiquitination process and its consequences. Here, we describe a new method to study this dynamic post-translational modification in intact human embryonic kidney cells. Using bioluminescence resonance energy transfer (BRET), we measured the ubiquitination of $\beta$-arrestin 2 , a regulatory protein implicated in the modulation of $G$ protein-coupled receptors. In addition to allowing the detection of basal and GPCR-regulated ubiquitination of $\beta$-arrestin 2 in living cells, real-time BRET measurements permitted the recording of distinct ubiquitination kinetics that are dictated by the identity of the activated receptor. The ubiquitination BRET assay should prove to be a useful tool for studying the dynamic ubiquitination of proteins and for understanding which cellular functions are regulated by this post-translational event.
\end{abstract}

Ubiquitination is a rapid and reversible post-translational modification that is involved in numerous aspects of biology. The 76-residue polypeptide ubiquitin fulfils essential functions in eukaryotes through its covalent attachment to other intracellular proteins $^{1,2}$. Historically, the best-characterized role for this modification is the targeting of proteins for degradation by the $26 \mathrm{~S}$ proteasome after the transfer of an ubiquitin chain of at least four units, referred to as polyubiquitination ${ }^{3}$. More recently, the addition of a single ubiquitin to one (monoubiquitination) or multiple (multiubiquitination) protein sites has been described ${ }^{4}$. In these cases, the post-translational modification serves as a reversible signal involved in a variety of key cellular functions including intracellular signaling, subcellular localization and protein-protein interactions.

Despite the growing interest in ubiquitination, inspired by the diversity of cellular functions that appear to be influenced by this process, the lack of tools for monitoring its dynamic regulation limits studies in this field. Western blot analysis using antibodies to ubiquitin allows the detection of protein ubiquitination. Although this assay can be used to determine whether a protein is ubiquitinated, it cannot be easily adapted to study the dynamic nature of the ubiquitination and deubiquitination cycles. To monitor the ubiquitination process in living cells, we took advantage of a bioluminescence resonance energy transfer (BRET) method, which allows real-time detection of protein-protein interactions in vivo. This technique is based on the nonradiative transfer of energy between a luminescent energy donor (here, Renilla luciferase, Rluc) and a fluorescent energy acceptor (here, green fluorescent protein, GFP). This is a system of choice for monitoring both constitutive and regulated intermolecular interactions because of the strict dependence on molecular proximity $(<100 \AA)$ between the donor and acceptor molecules for energy transfer ${ }^{5}$.

It was recently proposed that $\beta$-arrestin 2 ( $\beta$-arrestin), a versatile protein involved in the regulation of $\mathrm{G}$ protein-coupled receptor (GPCR) signaling ${ }^{6}$, is ubiquitinated in response to receptor activation $^{7}$. Based on the stability of their interaction with $\beta$-arrestin, two broad classes of GPCRs are distinguishable ${ }^{8,9}$ : GPCRs known as class A receptors (for example, the $\beta_{2}$-adrenergic receptor, $\beta_{2} \mathrm{AR}$ ) interact only transiently with $\beta$-arrestin after their activation, whereas activated class $B$ receptors (for example, the $V_{2}$-vasopressin receptor, $\mathrm{V}_{2} \mathrm{R}$ ) form stable complexes with $\beta$-arrestin. Recent findings ${ }^{10}$ are suggestive of a link between the dynamics of $\beta$-arrestin ubiquitination and the nature of its interaction with the activated receptor. A stable interaction correlates with sustained $\beta$-arrestin ubiquitination, whereas a transient interaction results in short-lived ubiquitination. Using $\beta$-arrestin as a model ubiquitinated protein, we report here that BRET allows monitoring of the changes in the ubiquitination state of a protein in real time.

\section{RESULTS}

Ubiquitination monitored by BRET in living cells

As ubiquitination results from the covalent attachment of ubiquitin to specific protein substrates, BRET should be adaptable to monitoring this post-translational modification in living cells. The covalent attachment of a GFP-ubiquitin fusion protein to a substrate fused to Renilla luciferase (Rluc) should bring the Rluc and GFP moieties within a BRET-permissive distance, resulting in a detectable BRET signal. To determine whether we could use BRET

\footnotetext{
${ }^{1}$ Département de Biochimie and Groupe de Recherche sur le Système Nerveux Autonome, Université de Montréal, C.P. 6128 Succursale Centre-Ville Montréal, Québec H3C 3J7, Canada. ${ }^{2}$ Laboratory of Functional Genomics, UPR CNRS 2580 CCIPE, 141 rue de la Cardonille, 34094 Montpellier, Cedex 05 France. ${ }^{3}$ These authors contributed equally to this work. Correspondence should be addressed to M.B. (michel.bouvier@umontreal.ca).

PUBLISHED ONLINE 18 NOVEMBER 2004; DOI:10.1038/NMETH722
} 


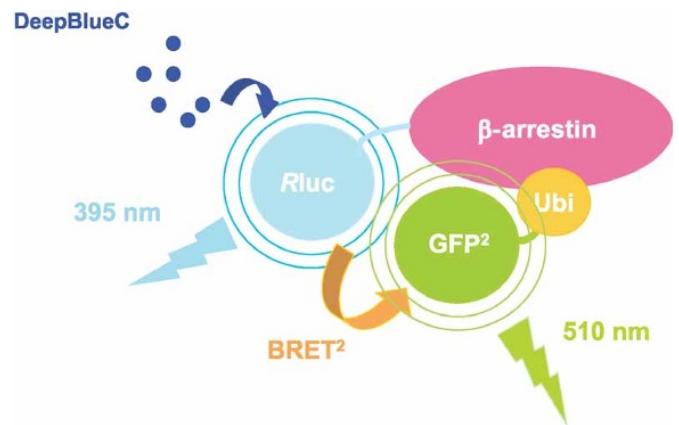

Figure 1 | Schematic representation of the $\beta$-arrestin ubiquitination BRET $^{2}$ assay. Upon degradation of its catalytic substrate (DeepBlueC coelenterazine), the Rluc fused to the $\mathrm{N}$ terminus of $\beta$-arrestin emits blue light with an emission peak at $395 \mathrm{~nm}$. If the $\beta$-arrestin is ubiquitinated, a nonradiative transfer of energy will occur between Rluc and the GFP2 fused to the N terminus of ubiquitin, resulting in re-emission of light with an emission peak at $510 \mathrm{~nm}$.

to study ubiquitination of $\beta$-arrestin, we designed N-terminally tagged Rluc- $\beta$-arrestin and GFP-ubiquitin (GFP'-Ubi) fusion protein constructs. The close proximity of several $\mathrm{GFP}^{2}$ moieties could cause quenching or interference phenomena that could lead to a decrease in the BRET signal, and indeed, we observed this (Supplementary Fig. 1 online). To limit the formation of poly $\left(\mathrm{GFP}^{2}-\mathrm{Ubi}\right)$ chains $^{11}$, we created a mutant $\mathrm{GFP}^{2}$-Ubi protein by changing Lys48 and Lys63, the two residues through which linkages occur, to alanine residues.

To initiate the energy transfer between Rluc and $\mathrm{GFP}^{2}$ in cells coexpressing Rluc- $\beta$-arrestin and $\mathrm{GFP}^{2}-\mathrm{Ubi}$, we used the luciferase substrate DeepBlueC coelenterazine, which readily crosses the plasma membrane. Hydrolysis of DeepBlueC coelenterazine leads to the emission of light with a spectrum overlapping the excitation spectrum of $\mathrm{GFP}^{2}$, thus allowing $\mathrm{BRET}^{2}$ (Fig. 1). Coexpression of a constant level of Rluc- $\beta$-arrestin with increasing concentrations of $\mathrm{GFP}^{2}$-Ubi led to an increase in the BRET ${ }^{2}$ signal (Fig. 2a). The energy transfer increased hyperbolically as a function of the GFP2 Ubi expression level, indicative of a specific interaction between the two proteins ${ }^{12}$. To confirm that the signal specifically reflects the ubiquitination process, we used either a $\mathrm{GFP}^{2}$-tagged ubiquitin mutant that is unable to take part in the ubiquitination process as a result of the replacement of its last two glycines by alanine residues $\left(\mathrm{GFP}^{2}-\mathrm{Ubi}_{\mathrm{AA}}\right)$ or $\mathrm{GFP}^{2}$ alone as negative controls. Coexpression of Rluc- $\beta$-arrestin with $\mathrm{GFP}^{2}-\mathrm{Ubi}_{\mathrm{AA}}$ or $\mathrm{GFP}^{2}$ led to weaker signals that increased linearly rather than hyperbolically with the increase in the fluorescence/luminescence ratio, most likely reflecting random collision (bystander BRET) between Rluc- $\beta$-arrestin and these two $\mathrm{GFP}^{2}$ constructs. Western blot analysis of lysates from cells expressing $\mathrm{GFP}^{2}$, $\mathrm{GFP}^{2}-\mathrm{Ubi}_{\mathrm{AA}}$ or $\mathrm{GFP}^{2}$-Ubi corroborated the lack of integration of $\mathrm{GFP}^{2}-\mathrm{Ubi}_{\mathrm{AA}}$ into proteins (Fig. 2b). The smeared band detected above $63 \mathrm{kDa}$ in lysates from cells expressing $\mathrm{GFP}^{2}$-Ubi most likely reflects ubiquitination of multiple proteins, and the sharp bands observed in lysates from cells expressing $\mathrm{GFP}^{2}-\mathrm{Ubi}_{\mathrm{AA}}$ probably correspond to monomeric and multimeric forms of the $\mathrm{GFP}^{2}$-tagged ubiquitin. The BRET ${ }^{2}$ signal detected between Rluc- $\beta$-arrestin and $\mathrm{GFP}^{2}$-Ubi did not result from the ubiquitination of the Rluc moiety itself, as no signal was detected when only $\mathrm{GFP}^{2}$-Ubi and Rluc were coexpressed (Fig. 2a). To determine whether the $\mathrm{GFP}^{2}$-Ubi protein can covalently modify $\beta$-arrestin, we immunoprecipitated Myc-tagged $\beta$-arrestin and detected GFP ${ }^{2}$-Ubi-immunoreactive species, which probably correspond to a ubiquitinated $\beta$-arrestin population (Fig. 2c). Taken together, these results demonstrated that energy transfer between $\mathrm{GFP}^{2}$-Ubi and Rluc- $\beta$-arrestin reflects a nonrandom clustering of these two proteins and the covalent ubiquitination of $\beta$-arrestin in vivo.

\section{Detection of agonist-induced ubiquitination}

In a recent study, ex vivo western blot analysis suggested that GPCR activation modulates $\beta$-arrestin ubiquitination ${ }^{7,10}$. To assess whether BRET can detect receptor-regulated ubiquitination in living cells, we monitored the transfer of energy between Rluc- $\beta$ arrestin and $\mathrm{GFP}^{2}$-Ubi in the presence of coexpressed $\beta_{2} \mathrm{AR}$ and a

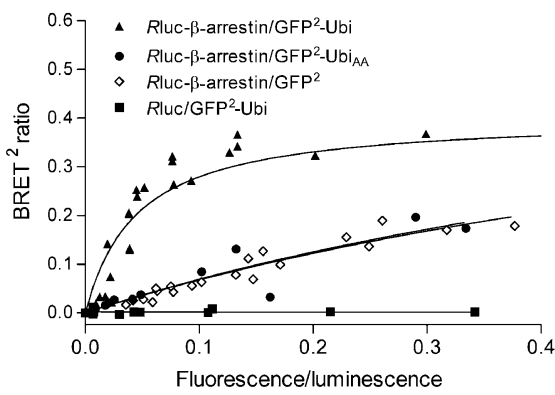

b

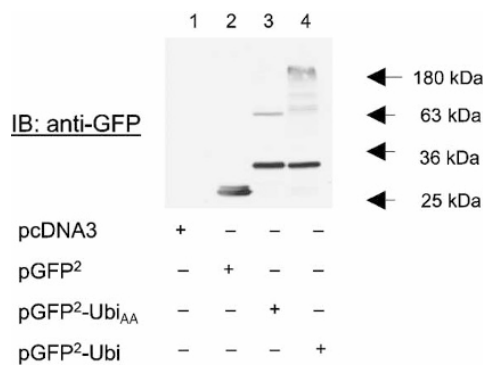

C

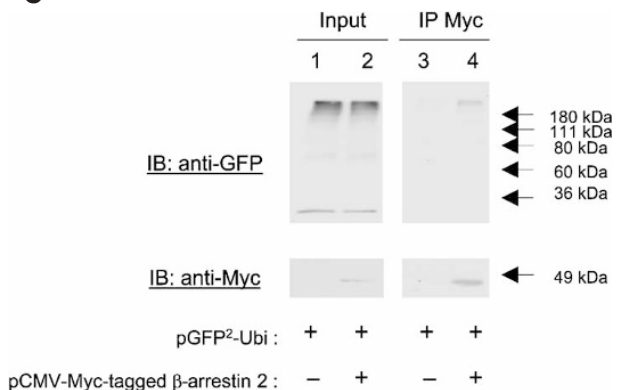

Figure 2 | Specific BRET ${ }^{2}$ signal between Rluc- $\beta$-arrestin and GFP²-Ubi. (a) HEK 293 cells were cotransfected either with constant concentrations of Rluc- $\beta$ arrestin, Myc-tagged $V_{2} R$ and HA-tagged $\beta_{2} A R$ DNA constructs and increasing concentrations of plasmids encoding GFP2-Ubi $(\boldsymbol{\Delta}), G F P^{2}-U b i i_{A A}(\bullet)$ or GFP2 $(\diamond)$, or with a constant concentration of Rluc and increasing concentrations of GFP²-Ubi DNAs ( $\mathbf{\square})$. The data shown represent pooled individual readings obtained from five to eight independent experiments. The curves were fitted using a nonlinear regression equation, assuming a single binding site (GraphPad Prism).

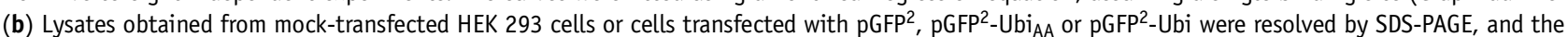
attachment of ubiquitin to proteins was assessed by western blot analysis using an antibody to GFP. Numbers on the right indicate molecular weight of protein markers. The blot shown is representative of two independent experiments. (c) Immunoprecipitation with an antibody to Myc was performed on lysates prepared from cells expressing $\mathrm{PGFP}^{2}$-Ubi or $\mathrm{pGFP}$-Ubi and pCMV-Myc-tagged $\beta$-arrestin. GFP2-Ubi protein associated with the immunoprecipitated $\beta$-arrestin was revealed as in $\mathbf{b}$ with an antibody to GFP, whereas the amount of $\beta$-arrestin immunoprecipitated was controlled with an antibody to Myc. 
a

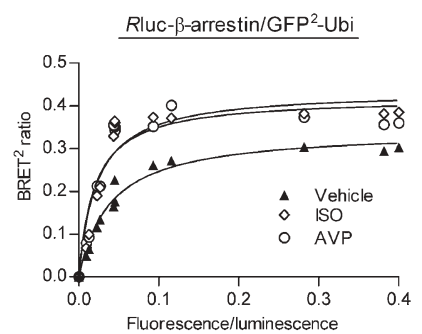

b

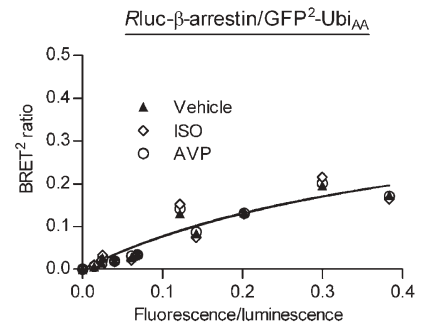

C

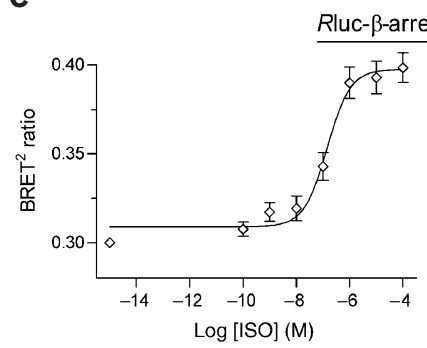

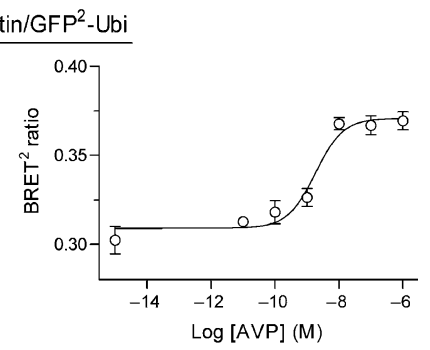

Figure 3 | Detection of GPCR-induced $\beta$-arrestin ubiquitination. (a,b) Cells cotransfected with a constant concentration of Rluc- $\beta$-arrestin, Myc-tagged $V_{2} R$ and HA-tagged $\beta_{2} \mathrm{AR}$ plasmids and increasing concentrations of GFP2-Ubi (a) or $\mathrm{GFP}^{2}-\mathrm{Ubi}_{\mathrm{AA}}(\mathbf{b})$ constructs were unstimulated $(\mathbf{\Delta})$ or stimulated with $10^{-5} \mathrm{M}$ ISO $(\diamond)$ or $10^{-6}$ M AVP $(\bigcirc)$ for $120 \mathrm{~s}$ before BRET measurement. DeepBlueC coelenterazine was added in the continuous presence of the agonists and the BRET measurements were taken $20 \mathrm{~s}$ after the addition of luciferase substrate. The data shown represent pooled individual readings obtained from three independent experiments. (c) Cells expressing the same construct as in a and displaying a GFP2-Ubi/Rluc- $\beta$-arrestin expression ratio of $0.1 \pm 0.005$ (assessed by measuring

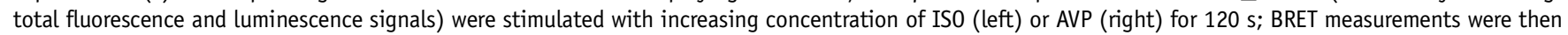
collected as in a. The curves shown represent the mean \pm s.e.m. of three independent experiments.

$\mathrm{V}_{2} \mathrm{R}$. Activation of each receptor with its selective agonist, isoproterenol (ISO) for the $\beta_{2} \mathrm{AR}$ and arginine-vasopressin (AVP) for the $\mathrm{V}_{2} \mathrm{R}$, substantially increased the BRET ${ }^{2}$ signal (Fig. 3a).

을 In contrast, ligands did not have an effect on the bystander BRET $^{2}$ observed between $\mathrm{GFP}^{2}-\mathrm{Ubi}_{\mathrm{AA}}$ and Rluc- $\beta$-arrestin (Fig. 3b), confirming the selectivity of the agonist-promoted ubiquitination observed with $\mathrm{GFP}^{2}$-Ubi. This increase in BRET ${ }^{2}$ signal was dose dependent, and the agonist concentrations yielding a half-maximal response $\left(\mathrm{EC}_{50}\right)$ were 140 and $1.8 \mathrm{nM}$ for ISO and AVP, respectively, consistent with the affinity of these ligands for their receptors (Fig. 3c).

\section{Concomitant detection of ubiquitination and recruitment}

It has been suggested that receptor activation may regulate the ubiquitination of $\beta$-arrestin and its recruitment to GPCRs ${ }^{10,13}$ in a coordinated fashion. To monitor these two events simultaneously in the same cells, we took advantage of the distinct spectral properties of two luciferase substrates that allow energy transfer with two different fluorescent proteins. Whereas the Rluc-catalyzed oxidation of DeepBlueC coelenterazine leads to BRET ${ }^{2}$ between Rluc and $\mathrm{GFP}^{2}$, the use of coelenterazine $\mathrm{h}$ leads to a distinct emission spectrum that promotes $\mathrm{BRET}^{1}$ between Rluc and a variant of GFP, the yellow fluorescent protein (YFP) ${ }^{14-16}$. We divided a culture of cells coexpressing Rluc- $\beta$-arrestin, $\mathrm{GFP}^{2}$-Ubi and $\mathrm{V}_{2} \mathrm{R}$-YFP into two samples. In one sample we monitored the $\beta$-arrestin ubiquitination by assessing the BRET $^{2}$ between

a

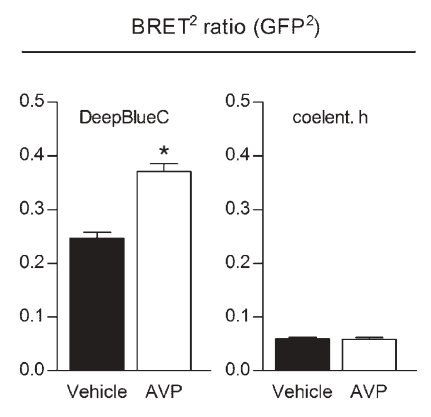

b

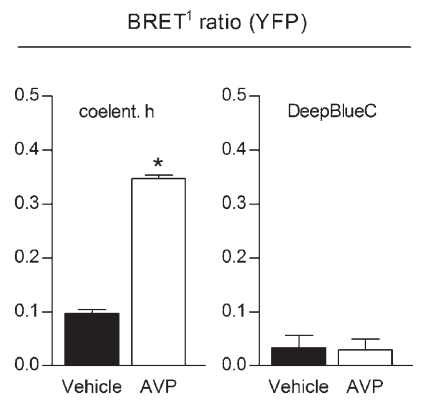

Rluc- $\beta$-arrestin and $\mathrm{GFP}^{2}-\mathrm{Ubi}$, and in the second sample we measured BRET ${ }^{1}$ between Rluc- $\beta$-arrestin and $\mathrm{V}_{2} \mathrm{R}$-YFP to evaluate the recruitment of $\beta$-arrestin to the receptor (Fig. 4). Stimulation with AVP resulted in an increase in both BRET ${ }^{2}$ and BRET $^{1}$ signals, reflecting concomitant ubiquitination (Fig. 4a) and recruitment of $\beta$-arrestin (Fig. $\mathbf{4 b}$ ). Each of the BRET signals detected was specific to the interaction monitored, as no substantial transfer of energy occurred between Rluc and $\mathrm{GFP}^{2}$ in the presence of coelenterazine $\mathrm{h}$ or between Rluc and YFP in the presence of DeepBlueC coelenterazine.

\section{Real time monitoring of distinct ubiquitination kinetics}

As described above, one can distinguish two GPCR classes based on their profile of interaction with $\beta$-arrestin. Because the ubiquitination state of $\beta$-arrestin seems intimately linked to its interaction profile with receptors ${ }^{10}$, we followed the kinetics of agonistpromoted $\beta$-arrestin ubiquitination in real time in cells coexpressing $\beta_{2} A R$ and $V_{2} R$. Both Iso and AVP induced rapid and similar increases in the BRET-detected ubiquitination of $\beta$-arrestin that reached a peak at 2 minutes after activation (Fig. 5). However, although it remained stable for at least $10 \mathrm{~min}$ after $\mathrm{V}_{2} \mathrm{R}$ activation (Fig. 5b,c), the BRET signal returned progressively toward basal values after activation of the $\beta_{2}$ AR (Fig. 5a,c). This reduction in BRET signal most likely reflects a deubiquitination process and not a degradation of $\beta$-arrestin. As BRET is a ratiometric measurement (GFP ${ }^{2}$ emission divided by Rluc emission), degradation of
Figure 4 | Concomitant detection of $\beta$-arrestin ubiquitination and its recruitment to the activated receptor. $(\mathbf{a}, \mathbf{b})$ Cells cotransfected with Rluc- $\beta$-arrestin, $V_{2} R-Y F P$ and GFP2-Ubi were stimulated with $1 \mu \mathrm{M}$ AVP or vehicle alone for 10 min. DeepBlueC coelenterazine $\left(B^{2} \mathrm{CT}^{2}, \mathrm{a}\right)$ or coelenterazine $\mathrm{h}\left(\mathrm{BRET}^{1}, \mathbf{b}\right)$ were then added in the continuous presence of the agonist and BRET measurements collected $20 \mathrm{~s}$ after the addition of the luciferase substrate to monitor $R$ luc- $\beta$-arrestin ubiquitination or its recruitment to $V_{2} R-Y F P$, respectively. Bar graphs shown represent the mean \pm s.e.m. of three independent experiments. The lack of cross-talk between BRET ${ }^{1}$ and BRET ${ }^{2}$ in the same samples was verified by measuring the BRET signal between Rluc- $\beta$-arrestin and GFP2-Ubi in the presence of coelanterazine $h$ for BRET ${ }^{2}$ and between Rluc- $\beta$-arrestin and $V_{2} R$-YFP in the presence of DeepBlueC for BRET ${ }^{1}$. Virtually no cross-talk signal was observed (right side of each panel). Statistical significance of the difference was assessed using paired Student's $t$-test. ${ }^{*} P<0.05$. 

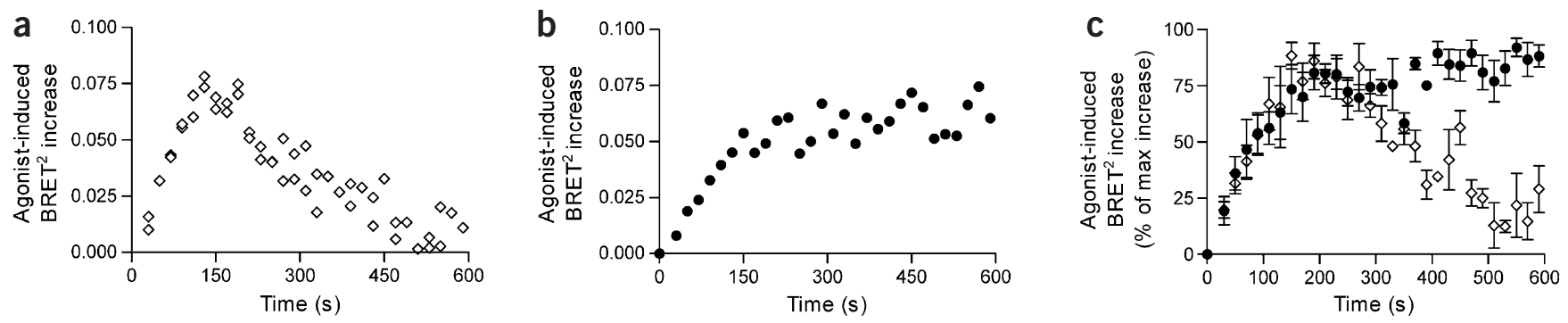

Figure 5 | Real-time assessment of receptor stimulated $\beta$-arrestin ubiquitination. Cells cotransfected with $R$ luc- $\beta$-arrestin, Myc-tagged $V_{2} R, H A$-tagged $\beta_{2} A R$ and GFP'-Ubi (GFP'-Ubi to Rluc- $\beta$-arrestin ratio of $0.1 \pm 0.005$ ) constructs, were incubated in the presence of DeepBlueC coelenterazine immediately before stimulation with $10^{-5} \mathrm{M}$ ISO $(\diamond ; \mathbf{a}, \mathbf{c})$ or $10^{-6} \mathrm{M} \operatorname{AVP}(\bullet ; \mathbf{b}, \mathbf{c})$. BRET $^{2}$ measurements were then collected immediately every $10 \mathrm{~s}$ in the continued presence of the agonists for $10 \mathrm{~min} .(\mathbf{a}, \mathbf{b})$ The real-time agonist-promoted increases in BRET are represented as the difference in the absolute BRET values obtained in the presence and in the absences of ISO or AVP. (c) Compilation of three independent experiments carried out as in $\mathbf{a}$ and $\mathbf{b}$. The data shown represent the mean \pm s.e.m. and are expressed in percentage of the maximal BRET ${ }^{2}$ signal obtained for each curve.

Rluc- $\beta$-arrestin should induce a decrease in luminescence and lead to an equivalent reduction in the transfer of energy to the acceptor without affecting the signal. These results, therefore, confirm that in living cells, the nature of the activated receptor determines the dynamics of $\beta$-arrestin ubiquitination.

\section{DISCUSSION}

The aim of this study was to adapt an energy transfer assay to the detection of protein ubiquitination in living cells. Using $\beta$-arrestin as a model, we demonstrated that BRET can specifically detect both basal and dynamically regulated ubiquitination processes. As compared to western blot analysis, the use of BRET presents several advantages: (i) because the BRET assay is carried out in living cells, it avoids the possible signal alterations that could result from cell lysis, protein solubilization or any purification steps before western blot analysis; (ii) the ability to follow the evolution of the BRET signal in real time allows one to capture the dynamic nature of the ubiquitination process; (iii) one can monitor changes in ubiquitination levels resulting from specific treatments in one population of cells using BRET, whereas western blot experiments require distinct cellular pools; and (iv) because of the ratiometric nature of the BRET technique, a reduction in signal truly represents a decrease in ubiquitination and does not result from the degradation of the ubiquitinated protein target.

As the BRET method is a proximity-based assay that was previously used to detect noncovalent protein-protein interactions, it was important to validate that BRET detected between Rluc- $\beta$ arrestin and $\mathrm{GFP}^{2}$-Ubi truly reflected the covalent attachment of the ubiquitin moiety. This was achieved by comparing the BRET signals obtained between Rluc- $\beta$-arrestin and either $\mathrm{GFP}^{2}$-Ubi, $\mathrm{GFP}^{2}-\mathrm{Ubi}_{\mathrm{AA}}$ or the GFP ${ }^{2}$ moiety alone. The BRET signal obtained between Rluc- $\beta$-arrestin and GFP $^{2}$-Ubi increased hyperbolically with increasing concentration of $\mathrm{GFP}^{2}$-Ubi before reaching a plateau once the covalent attachment of ubiquitin to Rluc- $\beta$ arrestin attained the maximal steady state. In contrast, the smaller, linear BRET signal obtained between Rluc- $\beta$-arrestin and $\mathrm{GFP}^{2}$ $\mathrm{Ubi}_{\mathrm{AA}}$ or $\mathrm{GFP}^{2}$ most likely resulted from bystander BRET ${ }^{12}$. Because it cannot be incorporated into proteins, the concentration of free $\mathrm{GFP}^{2}-\mathrm{Ubi}_{\mathrm{AA}}$ available for random collision should be equivalent to that reached when the $\mathrm{GFP}^{2}$ moiety alone is expressed, with both being much higher than that attained with $\mathrm{GFP}^{2}$-Ubi for equivalent $\mathrm{GFP}^{2}$ constructs. It follows that the bystander BRET component of the hyperbolic signal observed between Rluc- $\beta$-arrestin and GFP $^{2}$-Ubi should then be much smaller than that observed with $\mathrm{GFP}^{2}-\mathrm{Ubi}_{\mathrm{AA}}$ or $\mathrm{GFP}^{2}$. The lower signal detected between the Rluc moiety alone and $\mathrm{GFP}^{2}-\mathrm{Ubi}$ compared to that monitored between Rluc- $\beta$-arrestin and GFP 2 $\mathrm{Ubi}_{\mathrm{AA}}$ or $\mathrm{GFP}^{2}$ confirms this prediction. This makes $\mathrm{GFP}^{2}-\mathrm{Ubi}_{\mathrm{AA}}$ a stringent control that allows one to distinguish between proteinprotein interactions that are due to random collision and those due to covalent ubiquitination of a specific substrate.

Both BRET and immunoprecipitation approaches indicated that $\beta$-arrestin undergoes basal ubiquitination to a significant extent. These results are somewhat different from those of a published report $^{7,10}$ in which ubiquitination of $\beta$-arrestin was observed almost exclusively as a result of receptor activation. The specific reasons for this difference are not known, but it probably results from differences in the experimental conditions used, such as receptor expression level or the overexpression of exogenous ubiquitin protein.

An important aspect of the BRET-based method is its ability to detect dynamic changes in the ubiquitination state of a protein and to monitor changes over time. The ubiquitination BRET assay allowed us to monitor distinct kinetics of $\beta$-arrestin ubiquitination resulting from the activation of different GPCRs. Activation of the $\beta_{2} \mathrm{AR}$ induced a fleeting $\beta$-arrestin ubiquitination, whereas a longlasting ubiquitination of the regulatory protein was observed after activation of the $V_{2} R$. This is consistent with a previous study examining the ubiquitination status of $\beta$-arrestin at 1 and $15 \mathrm{~min}$ after receptor stimulation ${ }^{10}$. However, because the activated receptor recruits $\beta$-arrestin, the increase in ubiquitination BRET signal (Fig. 2a) could result from the ubiquitination of the receptor itself. This was not the case, as no BRET modulation occurred between $\beta_{2}$ AR-Rluc or $\mathrm{V}_{2}$ R-Rluc and GFP'2-Ubi after brief agonist stimulation (Supplementary Fig. 2 online). The data show that the ubiquitination BRET assay can faithfully reflect rapid and differential changes in the ubiquitination state of a specific substrate after specific stimuli.

The GFP2 ${ }^{2}$-Ubi construct used for the BRET experiments was designed to lack the two major sites involved in polyubiquitination in mammals (Lys48 and Lys63) to prevent the formation of GFP chains that could interfere with the energy transfer process. Despite the constraint that such a construct could impose on the system, ubiquitination of $\beta$-arrestin, which is believed to be 
polyubiquitinated $\mathrm{d}^{7,10}$, could be readily observed. This is not surprising given that the process of polyubiquitination permits the attachment of the GFP'2-Ubi through its terminal glycine either to $\beta$-arrestin targeted lysine(s) or to growing chains of ubiquitin. As the use of the GFP2-Ubi construct, lacking the polyubiquitination sites, could interfere with the properties of ubiquitinated proteins, we compared the ubiquitination BRET signal obtained with this construct to the signal obtained in the presence of a wild-type $\mathrm{GFP}^{2}$ Ubi protein $\left(\mathrm{GFP}^{2}-\mathrm{Ubi}_{\mathrm{WT}}\right)$. Although a specific hyperbolic BRET signal was detected between Rluc- $\beta$-arrestin and $\mathrm{GFP}^{2}-\mathrm{Ubi}_{\mathrm{WT}}$, it was lower than the one obtained with GFP'2-Ubi (Supplementary Fig. 1 online). Despite the lower absolute signal, similar BRET modulations were observed with both constructs after agonist stimulation (Supplementary Fig. 1 online). This indicates that $\mathrm{GFP}^{2}$-Ubi expression, per se, does not influence the nature of the results obtained but facilitates the detection of BRET signal changes. The fact that $\mathrm{GFP}^{2}-\mathrm{Ubi}_{\mathrm{WT}}$ could also be used in the ubiquitination BRET assay makes the method more broadly applicable.

Besides its usefulness in directly monitoring protein ubiquitination, the BRET assay offers the possibility of concomitant detection of additional protein interactions involving the ubiquitinated protein. Taking advantage of two spectrally distinct coelenterazines, we demonstrated the feasibility of such dual detection by monitoring both ubiquitination of $\beta$-arrestin and its recruitment to the $V_{2} R$ in the same cells.

In addition to shedding new light on the dynamics of $\beta$-arrestin ubiquitination in living cells, our study represents proof of the principle that resonance energy transfer approaches allow the monitoring of ubiquitin attachment in real time. BRET and possibly fluorescence resonance energy transfer (FRET) could be used to study the ubiquitination of various proteins whose activity and fate are regulated by this post-translational modification. Studies of the growing number of ubiquitin-like proteins that regulate diverse cellular functions through their covalent attachment to specific protein substrates could also benefit from advances in ubiquitination BRET assays.

\section{METHODS}

Plasmids. To produce a wild type $\mathrm{GFP}^{2}$-Ubi construct $\left(\mathrm{GFP}^{2}\right.$ $\mathrm{Ubi}_{\mathrm{WT}}$ ), we amplified the gene encoding human ubiquitin from a pQE32-Ubi plasmid and subcloned it into $\mathrm{pGFP}^{2}$-C1 (Perkin Elmer BioSignal). We then mutated by PCR lysines 48 and 63 in the ubiquitin moiety of the $\mathrm{GFP}^{2}-\mathrm{Ubi}_{\mathrm{WT}}$ construct into alanines (GFP'-Ubi). Finally, we constructed the $\mathrm{GFP}^{2}-\mathrm{Ubi}_{\mathrm{AA}}{ }^{17,18}$ by mutating by PCR the two C-terminal glycines 75 and 76 into alanines. We previously described pcDNA3.1-RLuc- $\beta$-arrestin, pcDNA3.1-hemagglutinin (HA)-tagged $\beta_{2} \mathrm{AR}$, pcDNA3.1-Myctagged $\mathrm{V}_{2} \mathrm{R}$ and pRK5- $\mathrm{V}_{2} \mathrm{R}-\mathrm{YFP} \mathrm{P}^{13,19-21}$. pCMV-Myc-tagged $\beta$-arrestin was a kind gift from S. Marullo's laboratory.

Cell culture and transfection. HEK293 cells were maintained in Dulbecco's modified Eagle's medium supplemented with $10 \%$ fetal bovine serum, $100 \mathrm{U} / \mathrm{ml}$ penicillin and streptomycin, $2 \mathrm{mM}$ L-glutamine (Wisent) and cultured at $37{ }^{\circ} \mathrm{C}$ in $5 \% \mathrm{CO}_{2}$. We transfected the cells $24 \mathrm{~h}$ after seeding using the calcium phosphate precipitation method ${ }^{22}$.

BRET measurement. Transfected cells were washed twice with PBS, detached in PBS with EDTA and resuspended in PBS with
$0.1 \%$ glucose. We determined the cell number by measuring protein concentration using the Dc protein assay kit (Bio-Rad). We obtained BRET measurements using two different sets of energy transfer partners. Energy transfer known as BRET ${ }^{2}$, between Rluc and $\mathrm{GFP}^{2}$, was determined after the addition of the Rluc substrate, DeepBlueC coelenterazine ${ }^{14}$ (Perkin Elmer), whereas BRET ${ }^{1}$, between Rluc and YFP, was assessed in the presence of another Rluc substrate, coelenterazine $h^{15,16}$.

BRET $^{2}$ monitoring of $\boldsymbol{\beta}$-arrestin ubiquitination. We distributed cells coexpressing Myc-tagged $\mathrm{V}_{2} \mathrm{R}$, HA-tagged $\beta_{2} \mathrm{AR}$, Rluc- $\beta$ arrestin and $\mathrm{GFP}^{2}$-Ubi constructs in white 96-well Microplates (Corning) at a density of 100,000 cells per well. We collected BRET $^{2}$ measurements $20 \mathrm{~s}$ after the addition of the luciferase substrate, DeepBlueC coelenterazine, at a final concentration of $5 \mu \mathrm{M}$. Readings were obtained using a modified TopCount NXT instrument (Packard Bioscience) that allows the sequential integration of the signals detected in the 370-450 and $500-530 \mathrm{~nm}$ ranges. The BRET ${ }^{2}$ signal corresponds to the ratio of the light emitted by the $\mathrm{GFP}^{2}(500-530 \mathrm{~nm})$ over the light emitted by the Rluc (370-450 nm). We corrected the values by subtracting the background ratio value detected when the Rluc constructs were expressed alone. For titration experiments, the BRET signal was plotted as a function of the total fluorescence signal over the total luminescence signal (see below), a ratio used as an index of the concentration of $\mathrm{GFP}^{2}-\mathrm{Ubi}$ (or $\mathrm{GFP}^{2}-\mathrm{Ubi}_{\mathrm{AA}}$ ) expressed.

BRET $^{1}$ monitoring of $\boldsymbol{\beta}$-arrestin recruitment to the $\mathrm{V}_{2} \mathrm{R}$ receptor. After dividing the cells coexpressing Rluc- $\beta$-arrestin, $\mathrm{GFP}_{2}$-Ubi and $\mathrm{V}_{2} \mathrm{R}$-YFP into two samples, we initiated BRET ${ }^{1}$ between Rluc$\beta$-arrestin and $V_{2} R$-YFP by the addition of $5 \mu \mathrm{M}$ coelenterazine $h$ (the recruitment of $\beta$-arrestin to the receptor). We collected the readings at the appropriate emission wavelengths after the addition of $1 \mu \mathrm{M}$ AVP using a Mithras LB 940 instrument (Berthold Technologies). We analyzed results as described above by calculating the ratio of the light emitted by the YFP $(510-550 \mathrm{~nm})$ to the light emitted by the Rluc $(460-500 \mathrm{~nm})$. We corrected the values by subtracting the background ratio value detected when the Rluc constructs were expressed alone.

Total fluorescence and luminescence measurements. We dispersed cells in white 96 well plates with clear bottoms (Costar) at a density of 100,000 cells per well ${ }^{12}$. We measured total fluorescence of cells in a Fluorocount (Packard Bioscience) using an excitation filter at 400 or $485 \mathrm{nM}$ and an emission filter at 510 or $530 \mathrm{nM}$ in the case of $\mathrm{GFP}^{2}$ or YFP, respectively. We then incubated cells for 10 min with coelanterazine $\mathrm{h}$ to measure the total luminescence using a LumiCount instrument (Packard Bioscience). In the case of fluorescence, we subtracted the background value detected in untransfected cells to give a specific fluorescence value. In the case of luminescence, where the background was negligible, we used absolute values.

Immunoblotting and immunoprecipitation. We solubilized cells expressing pcDNA3, GFP'- $\mathrm{Ubi}_{\mathrm{AA}}$ or $\mathrm{GFP}^{2}$-Ubi in lysis buffer A containing $50 \mathrm{mM}$ HEPES (pH7.5), $0.5 \%$ Nonidet P-40, $250 \mathrm{mM}$ $\mathrm{NaCl}, 2 \mathrm{mM}$ EDTA, $10 \%$ (v/v) glycerol, $5 \mathrm{mM} \mathrm{N}$-ethyl maleimide, $1 \mathrm{mM}$ phenylmethylsulfonyl fluoride, $5 \mu \mathrm{g} / \mathrm{ml}$ leupeptin, $5 \mu \mathrm{g} / \mathrm{ml}$ 
aprotinin and $100 \mu \mathrm{M}$ benzamidine. After separation of protein on SDS-PAGE and transfer to nitrocellulose, we revealed the immunoreactive bands using an antibody to GFP (Clontech), a horseradish peroxidase-conjugated antibody (Amersham) and the Western Lightning chemiluminescence Reagent Plus (Perkin Elmer). For immunoprecipitation experiments, we used cells expressing or not expressing pCMV-Myc-tagged $\beta$-arrestin 2 and $\mathrm{pGFP}^{2}$-Ubi and solubilized them in buffer A for $30 \mathrm{~min}$ with rocking at $4{ }^{\circ} \mathrm{C}$. We then incubated soluble extracts with an antibody to Myc (9E10) की and protein $\mathrm{G}$-agarose beads overnight at $4{ }^{\circ} \mathrm{C}$. Bound extracts were resolved by SDS-PAGE and transferred to nitrocellulose. We performed western blot analysis with an antibody to GFP, as described above, to reveal basal $\beta$-arrestin ubiquitination, and used A14 antibody to Myc (Santa Cruz) to detect the presence of Myctagged $\beta$-arrestin after its immunoprecipitation.

Note: Supplementary information is available on the Nature Methods website.

\section{ACKNOWLEDGMENTS}

The authors are grateful to A. Vallée-Bélisle and S. Michnick for providing the plasmid pQE32-Ubi and to S. Marullo for providing the pCMV-Myc-tagged $\beta$-arrestin 2 plasmid. This work was supported by grants from the Canadian

Institute for Health Research (M.B.) and the Natural Science and Engineering Research Council of Canada (M.A.). J.P. holds a fellowship from the European

Molecular Biology Organization, M.A. holds a scholarship from the Fond de

Recherche en Santé du Québec and M.B. is a Canada Research Chair in Signal

Transduction and Molecular Pharmacology.

\section{COMPETING INTERESTS STATEMENT}

The authors declare that they have no competing financial interests.

\section{Received 16 August; accepted 20 0ctober 2004}

Published online at http://www.nature.com/naturemethods/

1. Ciechanover, A., Orian, A. \& Schwartz, A.L. The ubiquitin-mediated proteolytic pathway: mode of action and clinical implications. J. Cell Biochem. Suppl. 34, 40-51 (2000).

2. Passmore, L.A. \& Barford, D. Getting into position: The catalytic mechanisms of protein ubiquitylation. Biochem. J. 379 (Part 3), 513-525 (2004).

3. Hochstrasser, M. Ubiquitin-dependent protein degradation. Annu. Rev. Genet. 30, 405-439 (1996).

4. Schnell, J.D. \& Hicke, L. Non-traditional functions of ubiquitin and ubiquitinbinding proteins. J. Biol. Chem. 278, 35857-35860 (2003).

5. Eidne, K.A., Kroeger, K.M. \& Hanyaloglu, A.C. Applications of novel resonance energy transfer techniques to study dynamic hormone receptor interactions in living cells. Trends Endocrinol. Metab. 13, 415-421 (2002).

6. Wojcikiewicz, R.J. Regulated ubiquitination of proteins in GPCR-initiated signaling pathways. Trends Pharmacol. Sci. 25, 35-41 (2004).
7. Shenoy, S.K., McDonald, P.H., Kohout, T.A. \& Lefkowitz, R.J. Regulation of receptor fate by ubiquitination of activated beta 2-adrenergic receptor and beta-arrestin. Science 294, 1307-1313 (2001).

8. Oakley, R.H., Laporte, S.A., Holt, J.A., Barak, L.S. \& Caron, M.G. Association of beta-arrestin with $\mathrm{G}$ protein-coupled receptors during clathrin-mediated endocytosis dictates the profile of receptor resensitization. J. Biol. Chem. 274, 32248-32257 (1999).

9. Oakley, R.H., Laporte, S.A., Holt, J.A., Caron, M.G. \& Barak, L.S. Differential affinities of visual arrestin, beta-arrestin 1 , and beta-arrestin 2 for $\mathrm{G}$ proteincoupled receptors delineate two major classes of receptors. J. Biol. Chem. 275, 17201-17210 (2000).

10. Shenoy, S.K. \& Lefkowitz, R.J. Trafficking patterns of beta-arrestin and G proteincoupled receptors determined by the kinetics of beta-arrestin deubiquitination. J. Biol. Chem. 278, 14498-14506 (2003).

11. Patrick, G.N., Bingol, B., Weld, H.A. \& Schuman, E.M. Ubiquitin-mediated proteasome activity is required for agonist-induced endocytosis of GluRs. Curr. Biol. 13, 2073-2081 (2003).

12. Mercier, J.F., Salahpour, A., Angers, S., Breit, A. \& Bouvier, M. Quantitative assessment of beta 1- and beta 2-adrenergic receptor homo- and heterodimerization by bioluminescence resonance energy transfer. J. Biol. Chem. 277, 44925-44931 (2002).

13. Lavoie, C. et al. Beta $1 /$ beta 2 -adrenergic receptor heterodimerization regulates beta 2-adrenergic receptor internalization and ERK signaling efficacy. J. Biol. Chem. 277, 35402-35410 (2002).

14. Jensen, A.A., Hansen, J.L., Sheikh, S.P. \& Brauner-Osborne, H. Probing intermolecular protein-protein interactions in the calcium-sensing receptor homodimer using bioluminescence resonance energy transfer (BRET). Eur. J. Biochem. 269, 5076-5087 (2002).

15. Angers, S. et al. Detection of beta 2-adrenergic receptor dimerization in living cells using bioluminescence resonance energy transfer (BRET). Proc. Natl. Acad. Sci. USA 97, 3684-3689 (2000).

16. Xu, Y., Piston, D.W. \& Johnson, C.H. A bioluminescence resonance energy transfer (BRET) system: application to interacting circadian clock proteins. Proc. Natl. Acad. Sci. USA 96, 151-156 (1999).

17. Haas, A.L., Murphy, K.E. \& Bright, P.M. The inactivation of ubiquitin accounts for the inability to demonstrate ATP, ubiquitin-dependent proteolysis in liver extracts. J. Biol. Chem. 260, 4694-4703 (1985).

18. Wilkinson, K.D. \& Audhya, T.K. Stimulation of ATP-dependent proteolysis requires ubiquitin with the $\mathrm{COOH}$-terminal sequence Arg-Gly-Gly. J. Biol. Chem. 256 9235-9241 (1981).

19. Perroy, J., Adam, L., Qanbar, R., Chenier, S. \& Bouvier, M. Phosphorylationindependent desensitization of GABA(B) receptor by GRK4. EMBO J. 22, 3816-3824 (2003).

20. Morello, J.P. et al. Pharmacological chaperones rescue cell-surface expression and function of misfolded V2 vasopressin receptor mutants. J. Clin. Invest. 105, 887-895 (2000).

21. Terrillon, S. et al. Oxytocin and vasopressin V1a and V2 receptors form constitutive homo- and hetero-dimers during biosynthesis. Mol. Endocrinol. 17, 677-691 (2003).

22. Mellon, P., Parker, V., Gluzman, Y. \& Maniatis, T. Identification of DNA sequences required for transcription of the human alpha 1-globin gene in a new SV40 host-vector system. Cell 27, 279-288 (1981). 